Centro de Investigación en

Metabolismo, Ejercicio y Salud (CIEMETS), Departamento de Educación Física, Universidad de la Frontera, Región de la Araucanía. Temuco, Chile. aProfesor de Educación Física. ${ }^{\text {bNutricionista. }}$ 'MsC. dPhD.

Recibido el 7 de diciembre de 2016, aceptado el 31 de mayo de 2017.

Correspondencia a: Erik Díaz B.

Centro de Investigación en Metabolismo, Ejercicio y Salud (CIEMETS).

Departamento de Educación Física, Universidad de la Frontera, Región de la Araucanía, Chile. erick.diaz@ufrontera.cl

\section{Entrenamiento interválico de alta intensidad contribuye a la normalización de la hipertensión arterial}

\author{
MARÍA ANGÉLICA OLEA, ${ }^{\mathrm{a}, \mathrm{c}}$, RODRIGO MANCILLA ${ }^{\mathrm{a}, \mathrm{c}}$, \\ SERGIO MARTÍNEZ ${ }^{\mathrm{a}, \mathrm{c}}$, ERIK DÍAZ ${ }^{\mathrm{b}, \mathrm{c}, \mathrm{d}}$
}

\section{Effects of high intensity interval training on blood pressure in hypertensive subjects}

Background: Exercise training may reduce blood pressure. Aim: To determine the effects of a high intensity interval training (HIIT) exercise protocol on systolic and diastolic blood pressure in hypertensive subjects. Material and Methods: Eleven men and 27 women aged $46.4 \pm 9.8$ years were divided in two groups according to their blood pressure. Sixteen were classified as normotensive and 22 as hypertensive. All attended an exercise program with 3 sessions per week for a total of 24 sessions. Each session consisted of one minute of intense exercise performed on a stationary bike, followed by an inactive pause lasting two minutes. This cycle was repeated 10 times and it was thus called $1{ }^{*} 2{ }^{*} 10$. Blood pressure, weight $(\mathrm{kg})$ and body fat were assessed. Results: In the hypertensive group, there was a significant reduction in systolic blood pressure from $145.4 \pm$ 9.0 to $118.3 \pm 15.6 \mathrm{~mm} \mathrm{Hg}(p<0.05)$. No significant change was observed in diastolic blood pressure $(84.9 \pm 3.9$ and $85.8 \pm 17.6 \mathrm{mmHg}$. Thus, there was a mean reduction in systolic pressure of $27.7 \pm 18.9 \mathrm{mmHg}$. Therefore, $73 \%$ of patients achieved systolic pressures within normal range, without medication. Conclusions: The $1{ }^{*} 2 * 10$ exercise method is effective to improve and restore normal blood pressure in persons with hypertension in a period of two months and 24 sessions.

(Rev Med Chile 2017; 145: 1154-1159)

Key words: Blood Pressure, Vasodilation, Vasoconstriction; Exercise; Hypertension.
L

as principales estrategias terapéuticas en hipertensión arterial (HTA) son el tratamiento farmacológico, la reducción de peso, la disminución de la ingesta de sodio y el ejercicio físico, prescritos de manera independiente o en conjunto. Sin embargo, todas ellas, rara vez logran normalizar los valores de presión arterial en pacientes hipertensos ${ }^{1}$. Algunos ejercicios físicos pueden tener efectos hipertensores o hipotensores agudos, no obstante, lo que realmente importa es lograr la adaptación crónica del sistema cardio- vascular central y periférico ${ }^{2,3}$. En este contexto, se han reportado importantes disminuciones de la presión arterial sistólica (PAS) y diastólica (PAD) en sujetos sedentarios, en respuesta a la práctica de ejercicios de sobrecarga y otras modalidades ${ }^{3,4}$, tanto en sujetos normotensos como hipertensos ${ }^{3,5}$. Interesantemente, estas reducciones en la presión arterial difieren en cuanto a su magnitud y duración según el tipo de ejercicio realizado (fuerza o pesas, aeróbicos), intensidad ${ }^{6} \mathrm{y}$ volumen de ejercicio $^{5,7}$. 
En términos de adaptación por efectos de ejercicio físico regular, se han dilucidado importantes modificaciones estructurales y funcionales en los vasos sanguíneos, tales como mayor elasticidad, disminución en el grosor de las arterias y reactividad vascular, entre otras ${ }^{8}$.

Dentro de los mecanismos hipotensores agudos del ejercicio está la secreción de óxido nítrico (ON) por las células endoteliales en respuesta al estrés por roce ${ }^{5,9}$. Otros factores circulantes son la producción del factor de crecimiento endotelial vascular (VEGF) e interleukina-15 (IL-15), que desencadenan el proceso de angiogénesis; mecanismo que facilitaría el flujo sanguíneo, produciendo una disminución de la presión arterial (PA). El ejercicio regular aumentaría la reactividad paracrina y la regulación del sistema simpático, mejorando la vasodilatación. Disminuiría, además, el grosor del vaso sanguíneo, aumentaría la reactividad del musculo liso y el lumen vascular ${ }^{11}$. Cabe destacar que todas estas adaptaciones parecen ser intensidad-dependientes del esfuerzo físi$\mathrm{co}^{10}$. Por ello, el objetivo del presente estudio fue determinar los efectos crónicos de un protocolo de ejercicio de alta intensidad intermitente (HIIT) sobre la presión arterial sistólica y diastólica en sujetos adultos normotensos e hipertensos.

\section{Método}

Se analizó la base de datos de los pacientes que asistieron al Centro de Investigación en Ejercicio Metabolismo y Salud (CIEMETS) de la Universidad de la Frontera. Se utilizó un muestreo no probabilístico de casos consecutivos seleccionándose 38 personas adultas (edad 46,4 \pm 9,8 años, 27 mujeres y 11 varones) que asistieron voluntariamente y por indicación médica al CIEMETS.

Todos ellos padecían alteraciones en el metabolismo oxidativo de grasas, tanto en reposo como en ejercicio, y respondían a los siguientes criterios de inclusión: tener sobrepeso u obesidad, no haber utilizado fármacos antihipertensivos ya fuera por consentimiento del médico tratante $o$ que el paciente no seguía el tratamiento farmacológico indicado y haber asistido a 24 sesiones ininterrumpidas. Criterios de exclusión fue no cumplir con los criterios anteriores o poseer síndrome metabólico, correspondiente a más de dos enfermedades crónicas no transmisibles.

Según su diagnóstico inicial de presión arte- rial los sujetos fueron divididos en normotensos (Grupo N) $(\mathrm{n}=16)$ e hipertensos (Grupo HTA) $(\mathrm{n}=22)^{11}$.

Al inicio del estudio se evaluó la capacidad física máxima $\left(\mathrm{VO}_{2 \text { máx }}\right)$ mediante el test de Astrand modificado en bicicleta ergómetra y criterios previamente reportados ${ }^{12,13}$, la medición de $\mathrm{VO}_{2}$ máx se repitió al final de la intervención en 29 personas solamente. Para la evaluación de composición corporal se utilizó un bioimpedanciómetro tipo pie-pie (Tanita ${ }^{\circledR}$, China), previamente validado ${ }^{14}$.

Ambos grupos fueron sometidos a un protocolo de entrenamiento fisico HIIT, durante 2 meses, 3 veces/semana. El ejercicio se ejecutó en bicicleta estática (Oxford, BE2700 ${ }^{\circledR}$, Chile) con una resistencia que indujo el fallo muscular (incapacidad de seguir pedaleando) al cabo de un minuto, seguido de 2 min de descanso inactivo, repetido 10 veces y supervisados de manera personalizada. La velocidad de pedaleo fue $30-40 \mathrm{~km} / \mathrm{h}$ que se debió mantener durante el minuto de ejercicio.

Durante el período de intervención se evaluó la presión arterial sistólica y diastólica al inicio y al final de cada una de las sesiones de entrenamiento empleando un esfigmomanómetro digital y automático, marca Citizen ${ }^{\circledR}$ (Digital Blood Pressure Monitor CH-452, Japón). Las mediciones se realizaron en el brazo izquierdo descubierto, con el sujeto en posición sentado- reposo. La medición preejercicio se llevó a cabo luego de 4 a 5 min de reposo, mientras que la evaluación postejercicio se ejecutó al cabo de 2-3 min una vez finalizada cada una de las sesiones de ejercicio (esta última no es reportada en este artículo). Los valores de presión arterial son expresados como $\mathrm{mmHg}$ y con el fin de tener certeza en la comparación pre vs post intervención, se tomó el valor promedio de la PAS de cada sujeto en las sesiones 1-3 para representar el período pre vs el promedio de PA en las sesiones 22-24 para el post intervención. En ambos casos las mediciones fueron realizadas previo a la sesión de ejercicio. Respecto a la reproducibilidad de las mediciones de PAS y PAD, se midió con el mismo equipo en dos ocasiones, en el mismo brazo, separados al menos de $2 \mathrm{~min}$, a un grupo de 10 personas, arrojando un error $<4 \%$. (PAS: 4,3 mmHg y PAD: 2,4 mmHg).

Todos los procedimientos empleados respetaron las normas éticas de la declaración de Helsinki y habían sido aprobados previamente por el Comité de Etica Institucional. 
Tabla 1. Características de los pacientes al inicio y final de la intervención

\begin{tabular}{|c|c|c|c|c|c|c|}
\hline \multirow{3}{*}{$\begin{array}{l}\mathbf{n} \\
\text { Género }\end{array}$} & \multicolumn{2}{|c|}{ Normotensos } & \multicolumn{4}{|c|}{ Hipertensos } \\
\hline & $\begin{array}{c}\text { Inicio } \\
16\end{array}$ & $\begin{array}{c}\text { Final } \\
16\end{array}$ & \multirow{2}{*}{$\begin{array}{c}\mathbf{p}^{*} \\
-\end{array}$} & $\begin{array}{c}\text { Inicio } \\
22\end{array}$ & $\begin{array}{c}\text { Final } \\
22\end{array}$ & \multirow{2}{*}{$\begin{array}{c}\mathbf{p}^{*} \\
-\end{array}$} \\
\hline & $3(\mathrm{M}) 13(\mathrm{~F})$ & $3(\mathrm{M}) 13(\mathrm{~F})$ & & $8(\mathrm{M}) \quad 14(\mathrm{~F})$ & $8(\mathrm{M}) \quad 14(\mathrm{~F})$ & \\
\hline Edad & $44,7 \pm 8,3$ & $44,7 \pm 8,3$ & - & $48,0 \pm 11,4$ & $48,0 \pm 11,4$ & - \\
\hline Peso & $76,0 \pm 14,7$ & $75,2 \pm 15,0$ & NS & $88,5 \pm 14,5$ & $85,0 \pm 14,1$ & $<0,05$ \\
\hline Talla & $161,2 \pm 6,5$ & $161,2 \pm 6,5$ & - & $166,4 \pm 6,5$ & $166,4 \pm 6,5$ & - \\
\hline $\mathrm{VO}_{2 \text { máx }}(\mathrm{ml} / \mathrm{kg} / \mathrm{min})^{\#}$ & $23,6 \pm 6,0$ & $27,0 \pm 6,8$ & $<0,05$ & $22,5 \pm 6,0$ & $26,0 \pm 6,5$ & $<0,05$ \\
\hline IMC & $29,2 \pm 5,1$ & $28,9 \pm 5,3$ & NS & $32,0 \pm 4,9$ & $30,7 \pm 5,0$ & $<0,05$ \\
\hline Grasa (kg) & $28,3 \pm 9,8$ & $27,5 \pm 10,4$ & NS & $36,5 \pm 7,0$ & $32,6 \pm 6,9$ & $<0,05$ \\
\hline Grasa (\%) & $36,5 \pm 7,4$ & $35,9 \pm 8,2$ & NS & $41,7 \pm 4,7$ & $38,4 \pm 5,4$ & $<0,05$ \\
\hline PAS & $118,1 \pm 6,8$ & $116,1 \pm 4,6$ & NS & $145,4 \pm 5,0$ & $118,3 \pm 15,6$ & $<0,05$ \\
\hline PAD & $72,9 \pm 7,8$ & $73,0 \pm 6,6$ & NS & $84,0 \pm 3,9$ & $74,6 \pm 6,0$ & NS \\
\hline
\end{tabular}

Grupos son descritos como (N) normotenso y (HTA) hipertensión arterial. (PAS) presión arterial sistólica y (PAD) presión arterial diastólica (ambas previas al ejercicio); $\mathrm{p}^{*}$ cambios significativos $<0,05$. NS: no significativos. Valores son promedio \pm desviación estándar; ${ }^{*} \mathrm{VO}_{2 \text { máx }}: \mathrm{n}=14$ grupo $\mathrm{N}$ y $\mathrm{n}=15$ grupo HTA.

\section{Análisis de datos}

La estadística descriptiva muestra datos promedio \pm desviación estándar. Para el análisis estadístico se empleó el programa SPSS versión 19. Para establecer la distribución normal de las variables se utilizó la prueba de Shapiro-Wilks, lo que resultó ser la situación para todas ellas. Por consiguiente, se utilizó la prueba t Student para muestras pareadas con el fin de analizar el cambio individual pre-post intervención. La comparación entre grupos se hizo mediante t Student para muestras independientes. La significación estadística de las diferencias se estableció con un $\mathrm{p}<0,05$.

\section{Resultados}

Ambos grupos fueron comparables al inicio de la intervención en todas las variables (Tabla 1), excepto en PAS y PAD $(p<0,05)$. Al comparar las variables evaluadas dentro de cada grupo en el período pre vs post intervención; no se observan variaciones en el grupo $\mathrm{N}$, a excepción del $\mathrm{VO}_{2 \text { máx }}$ donde hubo una aumento significativo al final del estudio $(\mathrm{p}<0,05)$, logrando alcanzar los valores promedio esperados según edad y sexo. A la vez, hubo disminución de peso y grasa corporales que no alcanzaron significación estadística. En contraste, el grupo HTA mostró una mejoría significativa del peso, $\mathrm{VO}_{2 \text { máx }}, \mathrm{IMC}$, grasa corporal y
PAS ( $\mathrm{p}<0,05)$. La PAD no experimentó cambios significativos en ninguno de los grupos, encontrándose dentro de los valores normales en todos los casos desde el inicio del estudio.

La Figura 1 muestra la variación de la presión arterial sistólica de los 22 sujetos del grupo HTA

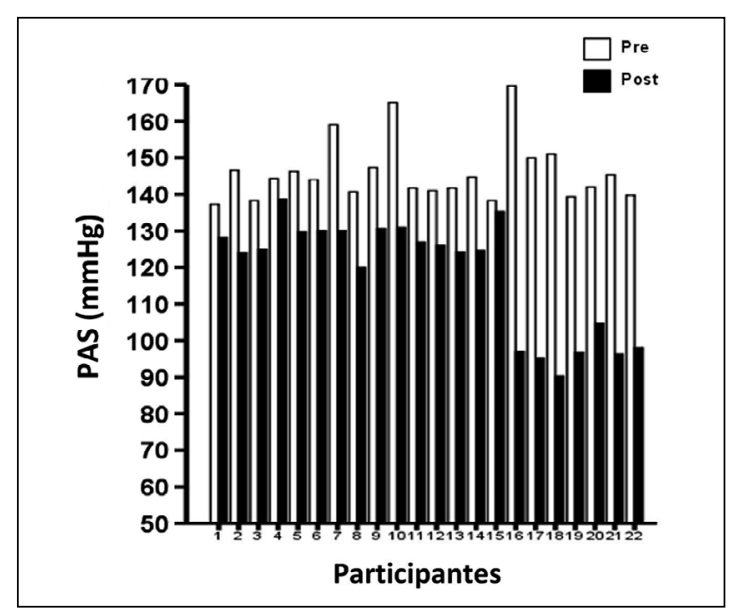

Figura 1. Variación de la presión arterial sistólica en cada sujeto previo al ejercicio en el grupo HTA. "Pre": corresponde a los valores promedio de las sesiones 1-3 vs "Post": sesiones 22-24 de la intervención, en ambos casos las mediciones fueron realizadas previo al ejercicio. Variables se presentan como (PAS) presión arterial sistólica, (Pre) inicio de la intervención, (Post) final de la intervención de ejercicio $(p<0,05)$. Delta promedio $27,7 \pm 18,9 \mathrm{mmHg}$. 
al inicio vs postintervención, disminuyendo en promedio 27,7 $\pm 18,9 \mathrm{mmHg}, \mathrm{p}<0,05$.

El diagnóstico de la PAS y sus variaciones durante la intervención en el grupo HTA se muestran en la Tabla 2 comparados al inicio, mitad y término de la intervención. Destaca que de los 22 pacientes con HTA sólo 6 permanecen con dicha condición luego de 11-13 sesiones y ninguno al finalizar la intervención. Al final de la intervención, la tasa de normalización de la PAS fue de $73 \%$ y la compensación se logró en el 100\%.

La Figura 2 muestra la disminución progresiva en la PAS promedio del grupo calculado para cada sesión en los 22 sujetos del grupo HTA, previo al

Tabla 2. Distribución del grado de presión arterial sistólica en el grupo HTA, clasificada de acuerdo a MINSAL, en tres etapas de la intervención

\begin{tabular}{|lcccc|}
\hline & $\begin{array}{c}\text { PAS } \\
(\mathbf{m m H g})\end{array}$ & $\begin{array}{c}\text { Inicio } \\
\left(\mathbf{1 - 3}^{\mathbf{a}} \text { sesión) }\right.\end{array}$ & $\begin{array}{c}\text { Medio } \\
\left(\mathbf{1 1 - 1 3 ^ { \mathbf { a } }} \text { sesión) }\right.\end{array}$ & $\begin{array}{c}\text { Final } \\
\left(\mathbf{2 2 - 2 4 ^ { \mathbf { a } }} \text { sesión) }\right.\end{array}$ \\
\hline HTA & $>140$ & 22 & 6 & 0 \\
PRE HTA & $130-139$ & 0 & 11 & 6 \\
Normal & $120-129$ & 0 & 4 & 8 \\
Optima & $<120$ & 0 & 1 & 8 \\
& & \% Normalización & $23 \%$ & $73 \%$ \\
& & \% Pre HTA & $50 \%$ & $27 \%$ \\
\hline
\end{tabular}

Grupos son descritos como (HTA): hipertensos; (PRE HTA): prehipertensos; (Normal): normotensos-altos y (Sanos): normotensos. PAS = presión arterial sistólica. "Inicio": promedio de las mediciones de PAS previo a las sesiones 1a-3a, "Medio": promedio de las mediciones de PAS previo a las sesiones $11^{\mathrm{a}}-13^{\mathrm{a}}$ "Final": promedio de las mediciones de PAS previo a las sesiones $22^{\mathrm{a}}-24^{\mathrm{a}} ; \mathrm{p}^{*}$ cambios significativos $<0,05$. NS: no significativos. "Normalización" corresponde al logro de PAS $<130 \mathrm{mmHg}$. "Prehipertensión" a PAS 130-139 mmHg (Orientaciones Técnicas Metas Sanitarias 2017, Subsecretaría de Redes Asistenciales MINSAL, septiembre 2016).

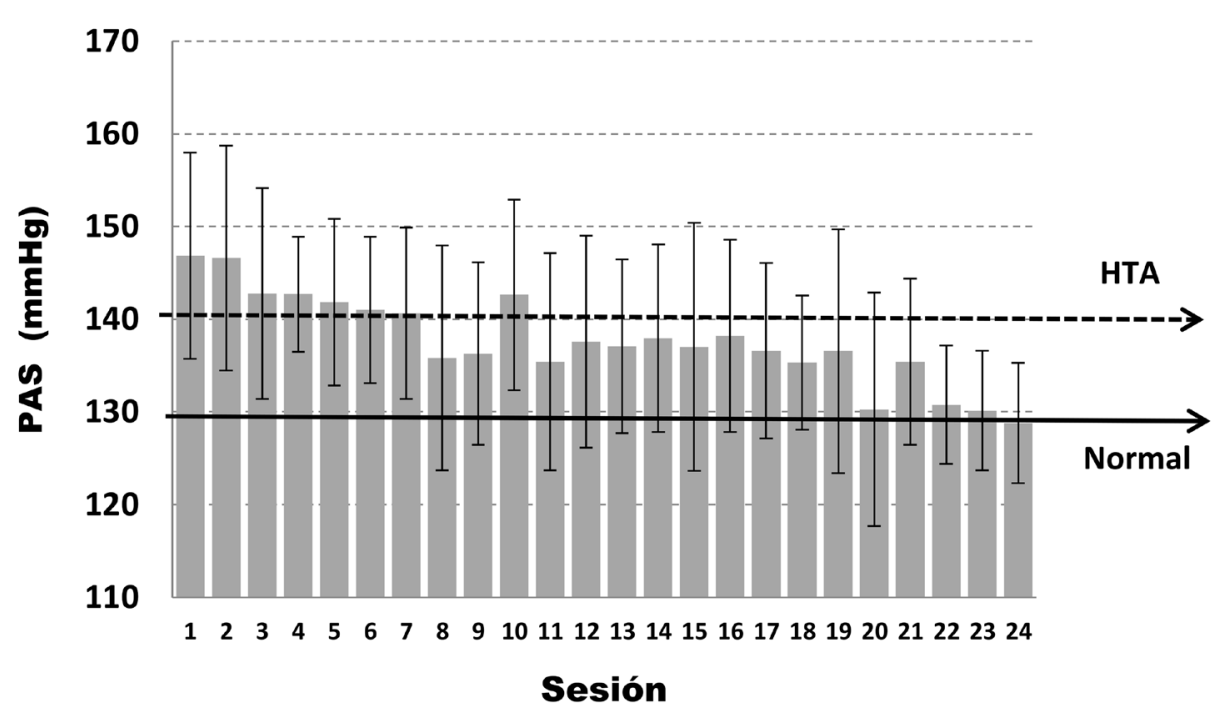

Figura 2. Variación promedio de la PAS en el grupo HTA, sesión a sesión, medición efectuada previo al ejercicio físico. Variables se presentan como (PAS) presión arterial sistólica. (....) Línea discontinua "HTA" indica límite de "hipertensión arterial". (__ Línea continua "Normal" incluye valores límites para "normal y normal alto"11. 
ejercicio. Destaca una tendencia en promedio a la reducción desde las primeras 2 sesiones, bajando a valores cercanos a la PAS de normalización $(<130 \mathrm{mmHg})$ entre las sesiones $20^{\mathrm{a}}-24^{\mathrm{a}}$.

\section{Discusión}

El modelo de intervención con ejercicio de HIIT en pacientes hipertensos contribuyó a normalizar la PAS de $73 \%$ de ellos y a compensar a $100 \%$ en un período de 2 meses. Lo anterior, sin interacción farmacológica durante la investigación. Esto concuerda con otro estudio, que empleó la misma metodología de ejercicio, pero con una duración de 6 semanas, la adición de ejercicios de sobrecarga y el uso de fármacos; logró compensar la PAS de $91 \%$ de los pacientes diabéticos/ hipertensos (DM2/HTA) y de $87 \%$ de pacientes con hipertensión arterial aislada (HTA). En el mismo estudio, cuando se utilizó solo fármacos sin ejercicio, se logró compensar a 33 y $25 \%$ de los casos DM2/HTA e HTA, respectivamente 7 . Desafortunadamente, no se menciona el porcentaje de pacientes que logra normalizar la presión arterial.

Boutcher y cols ${ }^{15}$, resumen las investigaciones sobre metodologías HIIT y sus efectos en PA, mostrando disminuciones de la PAS entre -2 y -12 mmHg o ausencia de cambios. Dichos resultados se obtienen empleando diversas metodologías de HIIT, con o sin empleo de fármacos. El único factor en común entre los estudios es la alta intensidad y la intermitencia (ejercicio-descanso), pero varían sustancialmente en el tiempo de ejercicio y la modalidad de descanso (activo-inactivo). Los resultados anteriores contrastan con el presente estudio, donde se logran disminuciones de 27,7 \pm $18,9 \mathrm{mmHg}$ en un período de tiempo de intervención similar, sin empleo de fármacos. Tan exitosos resultados replican los de un estudio pionero en Chile $^{16}$, que empleando ejercicio continuo de moderada intensidad por 30 min durante 3 meses en pacientes con HTA, sin medicación, logró disminuciones promedio de $-21,0 \pm 2,5 \mathrm{mmHg}$.

Otros programas de ejercicio denominados aeróbicos para el tratamiento de la HTA han logrado reducciones de PAS cuya importancia biológica es moderada, excepto el estudio antes mencionado $^{18}$. Por ejemplo, Punia y cols ${ }^{17}$, en una revisión sistemática de los estudios realizados en India en pacientes con HTA tratados con medicamentos antihipertensivos lograron -3 a -6 $\mathrm{mmHg}$. Nemoto y cols ${ }^{18}$ produjeron una reducción máxima de $-10 \mathrm{mmHg}$ en el grupo de caminata a elevada intensidad, al igual que Gunjal y cols ${ }^{19}$, quienes obtuvieron disminuciones promedio de $-4,5 \mathrm{mmHg}$. Ambas investigaciones no mencionan si hubo empleo de fármacos durante la intervención. Confirmando los efectos del ejercicio continuo en la PA, una revisión sistemática actual muestra reducciones promedio de -6 a -12 mmHg en la PAS, en grupos con HTA no tratada con fármacos ${ }^{20}$.

El logro de los resultados en la terapia de la HTA depende de la activación de los mecanismos involucrados en la disminución de la PA, que son principalmente paracrinos, fundamentalmente mediante la bradiquinina y el estrés por roce en los vasos sanguíneos ${ }^{21,22}$, que en conjunto con otros factores plasmáticos, aumentan la producción de ON endotelial, estimulan la angiogénesis, mejoran el lumen y el musculo liso del vaso sanguíneo, disminuyen el proceso inflamatorio vascular y la reactividad del sistema nervioso simpático. Todos ellos dan como resultado un significativo incremento en la reactividad vascular periférica y central ${ }^{12}$.

En conclusión, la HTA logró normalizarse mediante la contribución de un programa de ejercicio intermitente de alta intensidad, en sujetos hipertensos no medicados, en un período de 24 sesiones. A diferencia de otras modalidades de ejercicio intenso, el protocolo utilizado no presenta riesgos debido a la corta duración del estímulo seguido de descanso inactivo, que aún cuando la presión arterial sea muy elevada, ésta tiende a disminuir desde la primera serie de ejercicio terminando la sesión siempre con PA inferiores a las de su llegada al CIEMETS.

\section{Referencias}

1. Cano J, Ramírez R, Martínez C, Sade F, Salas A, Álvarez C. Interacción entre farmacoterapia hipotensiva y terapia con ejercicio físico requiere regulación farmacológica en pacientes hipertensos. Rev Med Chile 2016; 144: 152-61.

2. Aglony IM, Arnaiz GP, Acevedo BM, Barja YS, Márquez US, Guzmán AB, et al. Perfil de presión arterial e historia familiar de hipertensión en niños escolares sanos de Santiago de Chile. Rev Med Chile 2009; 137: 39-45.

3. Bode-Böger SM, Böger RH, Schröder EP, Frölich JC. 
Exercise increases systemic nitric oxide production in men. Eur J Prev Cardiology 1994; 1: 173-8.

4. Polito MD, Simão R, Senna GW, Farinatti P. Hypotensive effects of resistance exercises performed at different intensities and same work volumes. Rev Med Brasil 2003; 9: 74-7.

5. Blumenthal JA, Siegel WC, Appelbaum M. Failure of exercise to reduce blood pressure in patients with mild hypertension: Results of a randomized controlled trial. JAMA 1991; 266: 2098-104.

6. Álvarez C, Olivo J, Robinson O, Quintero J, Carrasco V, Ramírez-Campillo R, et al. Respuesta hipotensiva de la presión sistólica y diastólica a una sesión de ejercicio aeróbico en niños, adolescentes y adultos. Rev Med Chile 2013; 141: 1363-70.

7. Castañeda C, Layne JE, Muñoz-Orians L, Gordon PL, Walsmith J, Foldvari M, et al. A randomized controlled trial of resistance exercise training to improve glycemic control in older adults with type 2 diabetes. Diabetes Care 2002; 25: 2335-41.

8. Thijssen D, Cable T, Green D. Impact of exercise training on arterial wall thickness in humans. Clin Sci 2012; 122: 311-22.

9. Maroun MJ, Mehta S, Turcotte R, Cosio MG, Hussain SN. Effects of physical conditioning on endogenous nitric oxide output during exercise. J Appl Physiol 1995; 79: 1219-25.

10. Wisloff U, Stoylen A, Loennechen J, Bruvold M, Rognmo O, Haram P, et al. Superior cardiovascular effect of aerobic interval training versus moderate continuous training in heart failure patients. Circulation 2007; 115: 3086-94.

11. MINSAL. Guía clínica. Hipertensión Arterial Primaria o Esencial en Personas de 15 años y más. Santiago; 2010. p. 2-63.

12. Astrand PO. Chapter 8, Physical performance. In: Astrand P.O, Rodahl K, Dahl H, Stromme S. (eds). Textbook of work physiology: Physiological basis of exercise (4th ed). Champaign, IL, Human Kinetics 2003; p 237 -
72.

13. Mancilla R, Torres P, Álvarez C, Schifferli I, Sapunar J, Díaz E. Ejercicio físico interválico de alta intensidad mejora el control glicémico y la capacidad aeróbica en pacientes con intolerancia a la glucosa. Rev Med Chile 2014; 142: 34-9.

14. Jebb S, Cole T, Doman D, Murgatroyd P, Prentice A. Evaluation of the novel Tanita body-fat analyser to measure body composition by comparison with a four compartment model. Br J Nutr 2000; 83: 115-22.

15. Boutcher YN, Boutcher SH. Exercise intensity and hypertension: what's new? J Hum Hypertens (advance online publication), sept 8, 2016; doi: 10.1038/jhh.2016. 62.

16. Román O, Camuzzi AL, Villalón E, Klenner C. Pysical training program in arterial hypertension : A long- term prospective follow-up. Cardiology 1981; 67: 230-43.

17. Punia S, Kulandaivelan S, Singh V, Punia V. Effects of aerobic Exercise training on blood pressure in Indians: Systematic review. Int J Chronic Dis. (advance online publication) jul 17, 2016. doi: 10.1155/2016/1370148.

18. Nemoto K, Gen-Ho H, Masuki S, Okazaki K, Nose H. Effects of high-intensity interval walking training on physical fitness in middle-aged older people. Mayo Clin Proc 2007; 82: 803-11.

19. Gunjal S, Sinde N, Kazi A, Khatri S. Effect of aerobic interval training on blood pressure and myocardial function in hypertensive patients. Int J Pharma Sci Invest 2013; 2: 27-31.

20. Sabbahi A, Arena R, Elokda A, Phillips SA. Exercise and Hypertension: Uncovering the mechanisms of vascular control. Prog Cardiovasc Dis 2016; 59: 226-34.

21. Pyke KE, Tschakovsky ME. The relationship between shear stress and flow-mediated dilatation: implications for the assessment of endothelial function. J Physiol 2005; 568 (Pt 2): 357-69.

22. Irribarra V, Germain A, Cuevas A, Faúndez L, Valdés G. Disfunción endotelial como alteración primaria en las patologías vasculares. Rev Med Chile 2000; 128: 659-70. 